\title{
Triple Negative Breast Cancer - An Overview
}

\author{
Kartik Aysola"\#, Akshata Desai ${ }^{3 \#}$, Crystal Welch ${ }^{1}$, Jingyao Xu1 ${ }^{1}$, Yunlong Qin ${ }^{1}$, Vaishali Reddy ${ }^{1}$, Roland Matthews ${ }^{1}$, Charlotte Owens ${ }^{1}$, Joel \\ Okoli $^{2}$, Derrick J Beech ${ }^{2}$, Chandrika J Piyathilake ${ }^{4}$, Shyam P Reddy ${ }^{1}$ and Veena N Rao ${ }^{1}$
}

${ }^{1}$ Cancer Biology Program, Department of OB/GYN, Morehouse School of Medicine, Georgia Cancer Center for Excellence, Grady Health System, Atlanta, GA 30303, USA ${ }^{2}$ Department of Surgery, Morehouse School of Medicine, Grady Health System, Atlanta, GA 30303, USA

${ }^{3}$ Department of Internal Medicine, University of Buffalo, Erie County Medical Center, 462 Grider St, Buffalo NY 14215, USA

${ }^{4}$ Wallace Tumour Institute 420 D, 1824 6th Avenue South, University of Alabama at Birmingham, Birmingham AL 35294, USA

${ }^{*}$ contributed equally

\begin{abstract}
Triple Negative Breast Cancer (TNBC) is a heterogeneous disease that based on immunohistochemistry (IHC) is estrogen receptor $(E R)$ negative, progesterone receptor $(P R)$ negative and human epidermal growth factor receptor 2 (HER2) negative. TNBC is typically observed in young AA women and Hispanic women who carry a mutation in the BRCA1 gene. TNBC is characterized by a distinct molecular profile, aggressive nature and lack of targeted therapies. The purpose of this article is to review the current and future novel signalling pathways as therapeutic approaches to TNBC. Recent Identification of a new BRCA1 trafficking pathway holds promise in the future for the development of targeted therapies for TNBC.
\end{abstract}

Keywords: TNBC; BRCA1; CISPLATIN; EGFR; PARP; Hedgehog; NOTCH; BCL-2; UBC9; WNT/B-CATENIN

Triple Negative Breast Cancer (TNBC) is a subtype of breast cancer that based on immunohistochemistry (IHC) is estrogens receptor (ER) negative, progesterone receptor (PR) negative and human epidermal growth factor receptor 2 (HER2) negative [1]. TNBC is characterized by its unique molecular profile, aggressive nature, distinct metastatic patterns and lack of targeted therapies. It is estimated that out of the worldwide breast cancer burden, approximately 170,000 cases are TNBC and account for $\sim 10-20 \%$ of invasive breast cancers $[1,2]$.

\section{Molecular Profile and IHC Phenotype}

Breast cancers are typically classified into seven subtypes (3): luminal A (ER positive and histologic low grade), luminal B (ER positive and histologic high grade), HER2 overexpressing, basal-like (2 types - BL1 and BL2), immunomodulatory (IM), mesenchymal (M), mesenchymal stem-like (MSL) [3] and normal breast-like tumours [1]. Majority of the TNBC are the basal-like subtype, and many basallike breast cancers are triple negative; they are not equivalent in terms of gene expression signatures and IHC analysis [4]. Basal-like breast cancer is a classification based on gene expression profiling. Although they appear to be synonymous, there is up to $30 \%$ discordance between the two groups $[5,6]$. In addition to low expression of ER, PR and HER2, basal-like breast cancers are characterized by a high expression of CK5, CK14, caveolin-1, caix, p63, EGFR (Epidermal Growth Factor Receptor)/HER1, which reflects on the mammary gland basal/ myoepithelial cell component [7].

Additionally, several proteins integrally involved in DNA repair are aberrantly expressed in TNBC, which may have implications on sensitivity to chemotherapeutic agents, like, platinum-based drugs. High p53 IHC expression is common in basal-like breast cancer [8]. Several additional and targetable molecular pathways implicated in the pathogenesis of basal-like breast cancer include the mutagen activated protein (MAP) kinase pathway, the Akt pathway, and the poly ADPribose polymerase1 (PARP1) pathway [9].

\section{Association with BRCA1 Mutation status}

It has been observed that a high percentage of BRCA1- associated hereditary and sporadic breast cancers are triple negative and express a high proportion of basal like cytokeratins (CK5,14,17), as well as P-Cadherin and HER1/EGFR $[10,11]$. Gene expression studies support this association among patients with BRCA1 mutations that breast tumours tend to cluster within the basal like category [12].

\section{Epidemiology and Risk Factors}

Several large scale population based studies indicate that TNBC are up to three times more likely to occur among pre-menopausal women of African-American descent [13]. Certain epidemiologic studies like the Carolina Breast Cancer Study illustrated that as compared to the luminal a tumours, basal-like tumours were more likely to arise among women with early menarche, higher parity, younger age at full term pregnancy, and shorter duration of breast feeding, higher body mass index, and higher waist to hip ratio, especially among pre-menopausal patients [14]. Another study done by Bauer et al. [13] found that younger, non-Hispanic black and Hispanic women diagnosed with TNBC, had tumours that were more aggressive, and these women had poorer survival regardless of stage. In addition, non-Hispanic black women with late stage TNBC had the poorest survival of any comparable group [13].

\section{Clinical Characteristics}

TNBC is well known for its aggressive behaviour and is characterized by onset at a younger age, high mean tumour size, higher grade tumours and sometimes, a higher rate of node positivity [15]. Additionally, this group is also known for an early peak of recurrence between the first and third year after diagnosis, and more aggressive metastases which are more likely to occur in viscera particularly in the lungs and brain, and less likely to spread to the bone [16]. Based upon histological findings the majority of the triple negative breast cancers are of ductal origin; however, several other aggressive phenotypes also appear to be over

*Corresponding author: Veena N Rao, Professor and Co-director Cancer Biology Program, Gcc Distinguished Cancer Scholar, Department of Ob/Gyn, Morehouse School of Medicine, Georgia Cancer Center For Excellence, Rm 10c011, Grady Health System, 80 jesse hill jr. Drive, Atlanta, Georgia, 30303-3031, USA, Tel: 404489-9993; Fax: 404-489-9220; E-mail:vrao@msm.edu

Received March 11, 2013; Accepted April 15, 2013; Published April 19, 2013

Citation: Aysola K, Desai A, Welch C, Xu J, Qin Y, et al. (2013) Triple Negative Breast Cancer - An Overview. Genetics S2: 001. doi:10.4172/2161-1041.S2-001

Copyright: ( 2013 Aysola K, et al. This is an open-access article distributed under the terms of the Creative Commons Attribution License, which permits unrestricted use, distribution, and reproduction in any medium, provided the original author and source are credited. 
represented, including metaplastic, apocrine and adenoid cystic [17]. A histological study of basal-like tumours, all being ER/HER2 negative, yielded marked increase in mitotic count, geographic necrosis, pushing borders of invasion and stromal lymphocytic response [18].

\section{Prognosis}

An inferior prognosis in the basal-like breast cancer, as compared to the luminal type, has been uniformly demonstrated by a variety of studies [19]. Population based studies have also demonstrated similar results with reduced breast cancer specific survival among those with TNBC, as compared with the luminal subtype [14].

A recently reported Canadian series [15] evaluating prognosis in over 1,500 women, showed an increased likelihood of distant recurrence and death among women with triple negative breast cancer, as compared to the non triple negative disease. Studies have consistently shown that more aggressive visceral and soft tissue relapses are more common and bone relapses less common among those diagnosed with triple negative versus ER positive disease [20]. An estimated 15\% of all patients with breast cancer will develop brain metastasis. In a series of over 3,000 patients with brain metastasis arising from breast cancer treated from 1989 to 2006, multivariate analysis indicated that triple negative status was the greatest risk factor for the development of cerebral metastasis (odds ratio $=4.16 ; \mathrm{p}<0.001$ ), above that of HER2 positive status $(\mathrm{OR}=3.43 ; \mathrm{p}=0.005)$ [21]. In another study using patients with BRCA1 mutation, there was $82 \%$ complete pathologic response in those treated with Cisplatinum alone [22].

\section{Therapeutic Strategies}

Although triple negative breast cancers are associated with a generally poor breast cancer specific outcome, most are not resistant to chemotherapy. These patients have an extremely poor prognosis and relapse and die quickly. Several therapies are being developed that target specific biomarkers of TNBC or basal-like subtype [23]. These strategies include EGFR-targeted agents, androgen receptor targeted agents, anti-antigenic agents, and PARP inhibitors are offering an option in triple negative disease; however, their uses as of date are limited to clinical trials and more work is needed to identify targets that yield high therapeutic ratios [23]. TNBC with BRCA1 gene mutations may be more sensitive to agents that cause DNA damage, such as Cisplatin [24]. Other, more recent promising therapeutic targets for TNBC include the NOTCH, Hedgehog and Wnt/b-Catenin signalling pathways [2]. Studies have shown that these therapies alter the apoptotic pathway, inhibiting tumour progression [2].

\section{Chemotherapy}

Adjuvant chemotherapy has been shown to not only prolong disease-free survival in patients but overall survival as a whole; however, TNBC lacks the typical targeted receptors found in luminal or HER-2 disease and therefore cannot be treated with hormonal agents, such as SERMS, aromatise inhibitors or HER2 antagonists [25]. To combat this issue several neoadjuvant studies have been done which accentuated the relationship between chemo sensitivity and outcome, revealing proportionally higher sensitivity to anthracycline or anthracycline/taxane-based chemotherapy such as Doxorubicin and Cyclophosphamide (standard chemotherapeutic agents), for basal like/ER negative breast cancers as compared to the luminal subtype [26]. Highest response rates were noticed among those classified as basal like (85\%) and HER2 positive (70\%), as compared to luminal $(47 \%, p<0.0001)$. Despite initial chemo sensitivity, disease free survival $(\mathrm{p}=0.04)$ and overall survival $(\mathrm{p}=0.02)$ remained poorest among those with basal like and HER2 positive tumours, compared to the luminal subtype [26]. Although triple negative disease is highly responsive to Anthracycline/Taxane chemotherapy treatment, a high risk of relapse still remains if the tumour is not completely eradicated.

Additionally, pre-clinical and clinical studies indicate that tumours with BRCA1 dysfunction are sensitive to platinum agents such as Cisplatin, and Carboplatin which function by causing DNA damage and promote tumour cell apoptosis $[25,27]$. Studies have shown that p63, a family member of $\mathrm{p} 53$, is responsible for controlling a survival pathway that directly mediates Cisplatin sensitivity in TNBC; it therefore, can possibly be used as a biomarker to predict response to platinum therapy in triple negative breast cancer. However, this finding warrants further investigation and currently platinum's are not recommended for the adjuvant treatment of TNBC [25].

\section{EGFR Inhibitors}

EGFR expression is approximately seen in $60 \%$ of triple negative breast tumors, thus providing a reasonable targeted treatment approach [6]. A phase II trial evaluating the combination of Cetuximab (a chimeric monoclonal antibody targeting EGFR) and Carboplatin, weekly for 3 to 4 weeks reported a response rate of $18 \%$ and overall clinical benefit of $27 \%$ among 102 patients with advanced TNBC [28].

Another study evaluating the combination of Irinotecan and Carboplatin with or without Cetuximab reported response rates of $49 \%$ and $30 \%$ respectively, among 72 patients with pre-treated TNBC. EGFR inhibitor Panitumumab, when used in combination with standard chemotherapeutic agents for neoadjuvant therapy for inoperable TNBC was recently reported to have shown a pathological complete response rate of $65 \%$ [25]. Other studies have recently demonstrated that EGFR inhibitors when used in combination with Texans or platinum's may increase the efficacy of the other agents [25]. To date, the majority of data with EGFR inhibitors has generally been interpreted as negative.

\section{PARP Inhibitors}

PARP1, a gene that encodes an enzyme involved in the molecular events leading to cell recovery from DNA damage, when inhibited, leads to the accumulation of double-stranded DNA breaks. Cells deficient in BRCA1 and BRCA2 (required for normal homologous recombination), are exquisitely sensitive to PARP1 inhibition. Several PARP inhibitors Olaparib, Velaparib and PF-01367338 are currently in clinical trials and hold a promising future [25]. The study demonstrated a statistically significant $50 \%$ reduction in the risk of death; however, phase III trials failed to show statistically significant benefit for this combination, hence, the drug has been discontinued but some biomarker analysis is still underway to determine if a specific subset of patient may benefit from the drug $[16,28,29]$. This therefore highlights the need for continued research and clinical trials.

\section{Antiangiogenic Agents}

The antiangiogenic agent Bevacizumab, a monoclonal antibody that targets all forms of VEGF, has been evaluated in a number of large phase III trials as treatment of metastatic breast cancer [25]. The landmark study E2100 illustrated improvement in progressionfree survival (11.8 vs. 5.9 months, $\mathrm{HR}=0.60, p<0.001)$ when adding Bevacizumab to Paclitaxel chemotherapy compared with single-agent Paclitaxel alone in first-line treatment of metastatic disease [25]. This subsequently led to subgroup analysis that demonstrated similar progression free survival benefits in both patients with TNBC and non-TNBC with the use of Bevacizumab plus a taxane [25]. Currently, the BEATRICE trial is prospectively investigating this combination as 
adjuvant therapy in TNBC [25]. Additionally, several small-molecule inhibitors of the VEGF pathway appear to have activity in the subset of pre-treated triple-negative breast cancer and definitive studies showing overall survival benefit will be needed prior to re approval [30,31].

\section{Conclusion}

In conclusion, TNBC is a difficult and complex disease entity that is both confusing and frustrating for researchers, physicians and patients. To date there are multiple approaches attempting to improve care of triple negative breast cancer patients, including DNA damaging agents like platinum's, targeted EGFR and VEGF inhibitors, and, PARP inhibitors; however, none have been as clinically successful as anticipated and more targeted therapies need to be developed and explored. The Wnt/b-Catenin, NOTCH and Hedgehog signaling pathways are being considered as novel therapeutic targets for TNBC [2].

\section{Future Direction}

The key to developing targeted therapies for TNBC will depend on understanding the molecular mechanism for the development of these cancers. There are seminal studies by Hosey et al. [32] and by our group $[33,34]$ (unpublished results) on the possible molecular mechanisms of BRCA1 dysfunction which could result in ER negative breast cancers. Recently Ubc9, a sole SUMO-E2-conjugating enzyme for sumoylation was found to bind BRCA1 proteins and ferry it to the nucleus unlike disease associated mutant BRCA1 proteins [34]. Over expression of Ubc9 was observed in breast tumours and TNBC cell lines [34]. BRCA1 function-based cellular assays have been designed to detect loss of binding to Ubc9 by BRCA1 mutants (Patent number US 8,372,580). These assays not only predict the risk for developing TNBC but may also lead to the development of targeted therapies for hereditary and sporadic TNBC.

\section{References}

1. Perou CM (2011) Molecular stratification of triple-negative breast cancers. Oncologist 16 Suppl 1: 61-70

2. O'Toole SA, Beith JM, Millar EK, West R, McLean A, et al. (2013) Therapeutic targets in triple negative breast cancer. J Clin Pathol

3. Lehmann BD, Bauer JA, Chen X, Sanders ME, Chakravarthy AB, et al. (2011) Identification of human triple-negative breast cancer subtypes and preclinical models for selection of targeted therapies. J Clin Invest 121: 2750-2767.

4. Foulkes WD, Smith IE, Reis-Filho JS (2010) Triple-negative breast cancer. N Engl J Med 363: 1938-1948.

5. Bertucci F, Finetti P, Cervera N, Esterni B, Hermitte F, et al. (2008) How basal are triple-negative breast cancers? Int J Cancer 123: 236-240.

6. Nielsen TO, Hsu FD, Jensen K, Cheang M, Karaca G, et al. (2004) Immunohistochemical and clinical characterization of the basal-like subtype of invasive breast carcinoma. Clin Cancer Res 10: 5367-5374.

7. Rakha EA, Reis-Filho JS, Ellis IO (2008) Basal-like breast cancer: a critical review. J Clin Oncol 26: 2568-2581.

8. Troester MA, Herschkowitz JI, Oh DS, He X, Hoadley KA, et al. (2006) Gene expression patterns associated with p53 status in breast cancer. BMC Cancer 6: 276 .

9. Cleator S, Heller W, Coombes R (2007) Triple-negative breast cancer: Therapeutic options. Lancet Oncol 3: 235-244

10. Foulkes WD, Stefansson IM, Chappuis PO, Bégin LR, Goffin JR, et al. (2003) Germline BRCA1 mutations and a basal epithelial phenotype in breast cancer. J Natl Cancer Inst 95: 1482-1485.

11. Turner N, Tutt A, Ashworth A (2004) Hallmarks of 'BRCAness' in sporadic cancers. Nat Rev Cancer 4: 814-819.

12. Sorlie T, Tibshirani R, Parker J, Hastie T, Marron JS, et al. (2003) Repeated observation of breast tumor subtypes in independent gene expression data sets. Proc Natl Acad Sci USA 100: 8418-8423.
13. Bauer K, Brown M, Cress R, Parise CA, Caggiano V (2007) Descriptive analysis of estrogen receptor (ER)-negative, progesterone receptor (PR)-negative, and HER2-negative invasive breast cancer, the so-called triple-negative phenotype: A population-based study from the California cancer registry. Cancer 109: $1721-1728$

14. Carey LA, Perou CM, Livasy CA, Dressler LG, Cowan D, et al. (2006) Race, breast cancer subtypes, and survival in the Carolina Breast Cancer Study JAMA 295: 2492-2502.

15. Dent R TM, Pritchard KI, Hanna WM (2007) Triple-negative breast cancer: Clinical features and patterns of recurrence. Clin Cancer Res 13: 4429-4434

16. Criscitiello C, Azim HA Jr, Schouten PC, Linn SC, Sotiriou C (2012) Understanding the biology of triple-negative breast cancer. Ann Oncol 23 (supp 6); vi13-vi18

17. Reis-Filho JS, Milanezi F, Steele D, Savage K, Simpson PT, et al. (2006) Metaplastic breast carcinomas are basal-like tumours. Histopathology 49: 10-21.

18. Livasy CA, Karaca G, Nanda R, Tretiakova MS, Olopade OI, et al. (2006) Phenotypic evaluation of the basal-like subtype of invasive breast carcinoma. Mod Pathol 19: 264-271.

19. Sørlie T, Perou CM, Tibshirani R, Aas T, Geisler S, et al. (2001) Gene expression patterns of breast carcinomas distinguish tumor subclasses with clinical implications. Proc Natl Acad Sci USA 98: 10869-10874.

20. Smid M, Wang Y, Zhang Y, Sieuwerts AM, Yu J, et al. (2008) Subtypes of breas cancer show preferential site of relapse. Cancer Res 68: 3108-3114

21. Heitz F, Harter P, Traut A, Lueck HJ, Beutel B, et al. (2008) Cerebral metastases (CM) in breast cancer (BC) with focus on triple-negative tumors (abstract 1010). $\mathrm{J}$ Clin Oncol 26: 43s.

22. Byrski T, Gronwald J, Huzarski T, Grzybowska E, Budryk M, et al. (2010) Pathologic complete response rates in young women with BRCA1-positive breast cancers after neoadjuvant chemotherapy. J Clin Oncol 28: 375-379.

23. Penault F, Viale G (2012) Pathological and molecular diagnosis of triplenegative breast cancer: a clinical perspective. Ann Oncol 23: vi19-vi22.

24. Gonzalez-Angulo AM, Timms KM, Liu S, Chen H, Litton JK, et al. (2011) Incidence and outcome of BRCA mutations in unselected patients with triple receptor-negative breast cancer. Clinical Cancer Res 17: 1082-1089.

25. Joensuu H, Gligorov J (2012) Adjuvant treatments for triple-negative breast cancers. Ann Oncol 23 vi40-vi45.

26. Carey L, Dees E, Sawyer L, Gatti L, Moore DT, et al. (2007) The triple negative paradox: Primary tumor chemosensitivity of breast cancer subtypes. Clin Cancer Res 13:2329-2334.

27. (2011) Sanofi breast cancer drug flunks Phase III trial.

28. Carey LA, Rugo HS, Marcom PK (2009) EGFR inhibition with cetuximab added to carboplatin in metastatic triple-negative (basal-like) breast cancer. (Abstract 1009) J Clin Oncol

29. Taniguchi T, Tischkowitz M, Ameziane N, Hodgson SV, Mathew CG, et al (2003) Disruption of the Fanconi anemia-BRCA pathway in cisplatin-sensitive ovarian tumors. Nat Med 9: 568-574.

30. Abrams TJ, Murray LJ, Pesenti E, Holway VW, Colombo T, et al. (2003) Preclinical evaluation of the tyrosine kinase inhibitor SU11248 as a single agent and in combination with "standard of care" therapeutic agents for the treatment of breast cancer. Mol Cancer Ther 2: 1011-1021.

31. Burstein HJ, Elias AD, Rugo HS, Cobleigh MA, Wolff AC, et al. (2008) Phase II study of sunitinib malate, an oral multitargeted tyrosine kinase inhibitor, in patients with metastatic breast cancer previously treated with an anthracycline and a taxane. J Clin Oncol 26: 1810-1816.

32. Hosey AM, Gorski JJ, Murray MM, Quinn JE, Chung WY, et al. (2007) Molecular basis for estrogen receptor alpha deficiency in BRCA1-linked breast cancer. $J$ Natl Cancer Inst 99: 1683-1694.

33. Xu J, Watkins T, Reddy A, Reddy ES, Rao VN (2009) A novel mechanism whereby BRCA1/1a/1b fine tunes the dynamic complex interplay between SUMO-dependent/independent activities of Ubc9 on E2-induced ERalpha activation/repression and degradation in breast cancer cells. Int J Oncol 34: 939-949.

34. Qin Y, Xu J, Aysola K, Begum N, Reddy V, et al. (2011) Ubc9 mediates nuclear localization and growth suppression of BRCA1 and BRCA1a proteins. J Cell Physiol 226: 3355-3367. 